\title{
The distance to G59.7+0.1
}

\author{
Y. Xu ${ }^{1,2} \dagger$, M. J. Reid ${ }^{3}$, K. M. Menten ${ }^{1}$, A. Brunthaler ${ }^{1}$, \\ X. W. Zheng ${ }^{4}$ and L. Moscadelli ${ }^{5}$ \\ ${ }^{1}$ Max-Planck-Institute für Radioastronomie, Bonn, Germany \\ email: xuye@mpifr-bonn.mpg.de \\ ${ }^{2}$ Purple Mountain Observatory, Nanjing, China \\ ${ }^{3}$ Harvard-Smithsonian Center for Astrophysics, USA \\ ${ }^{4}$ Nanjing University, China \\ ${ }^{5}$ Arcetri Obs., Firenze, Italy
}

\begin{abstract}
We have measured the distance to the high-mass star-forming region G59.7+0.1, which harbors the far-infra-red source IRAS $19410+2336$. The distance is $2.20 \pm 0.11 \mathrm{kpc}$ and was determined by triangulation using Very Long Baseline Array (VLBA) observations of 12.2 $\mathrm{GHz}$ methanol masers phase-referenced to two compact extragalactic radio sources.
\end{abstract}

Keywords. ISM: molecules, Galaxy: kinematics and dynamics, Galaxy: structure.

\section{Introduction}

We are carrying out a large project to study the spiral structure and kinematics of the Milky Way (Xu et al. 2006). Here we report our results for G59.7+0.1, a very active star-forming region (Fig.1, left), which contains millimeter-wavelength dust continuum emission on multiple scales (Beuther \& Schilke 2004), including a dense hot core with an associated methanol maser emission and a weak centimeter-wavelength continuum sourcse (Beuther et al. 2002).

\section{Observations and Results}

We have conducted phase-referenced observations of G59.7+0.1 with the VLBA over 7 epochs in order to measure its relative position (using its methanol masers as astrometric targets) with respect to extragalactic radio sources. In Fig. 1 (right) we show an image from the the first epoch of a maser spot and of each of the background radio sources, which were phase-referenced relative to the strong maser emission. The QSOs as well as the maser spot are compact and appear dominated by a single component.

In order to provide the data needed to measure the parallax and proper motion, we fit Gaussian brightness distributions to the brightest maser spot and the two background radio sources for all seven epochs. In Fig. 2 we plot the positions of the maser spot relative to the two background radio sources. The results are:

Parallax: 0.454 \pm 0.022 mas (Distance: $2.20 \pm 0.11 \mathrm{kpc}$ )

Proper motion in R.A.: $-1.63 \pm 0.04$ mas $^{-1}$

Proper motion in Decl.: $-5.12 \pm 0.1$ mas $^{-1}$

The 3-D motion of G59.7+0.1 at its position in the Galaxy, assuming $R_{0}=8.5 \mathrm{kpc}$ and $\Theta_{0}=220 \mathrm{~km} \mathrm{~s}^{-1}$, is as follows:

In the direction of Galactic rotation: $215 \pm 10 \mathrm{~km} \mathrm{~s}^{-1}$;

Radial toward the Galactic Center: $+9 \pm 3 \mathrm{~km} \mathrm{~s}^{-1}$

$\dagger$ Present address: Auf dem Hügel 69 D-53121 Bonn, Germany. 

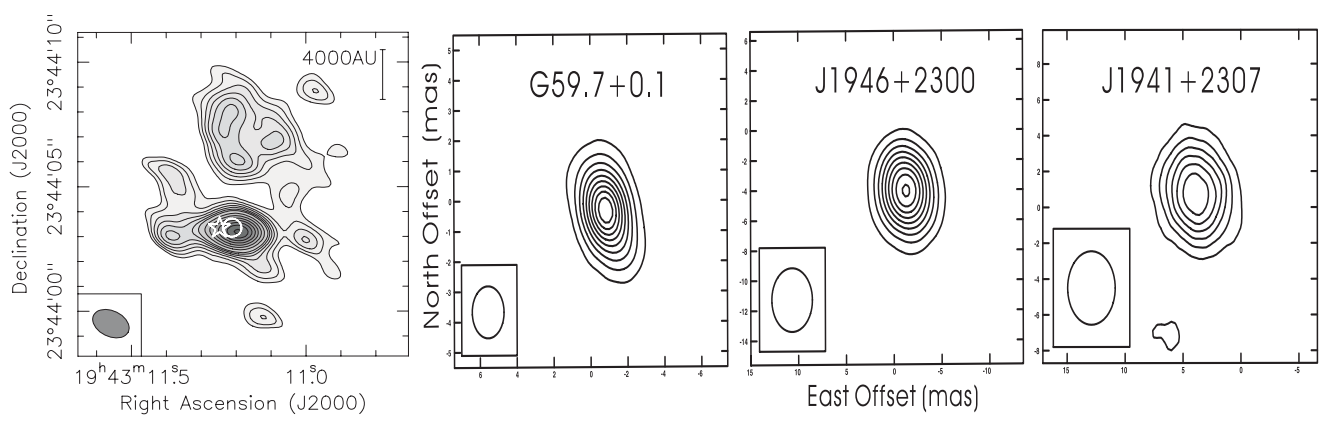

Figure 1. Left: The contours represent $230 \mathrm{GHz}$ mm continuum emission imaged with the IRAM Plateau de Bure interferometer by Beuther \& Schilke (2004). The circle marks the position of a compact, weak (1 mJy) $3.6 \mathrm{~cm}$ radio continuum source (from Beuther et al. 2002) and the star that of the $\mathrm{CH}_{3} \mathrm{OH}$ maser. The restoring beam of the interferometer observation is represented in the lower left part of the figure. Right: Synthesized images of the maser (left) and two background radio sources from the first epoch. The contour levels are multiples of $150 \mathrm{mJy}$ for G59.7+0.1, $2 \mathrm{mJy}$ for J1941+2307 and $10 \mathrm{mJy}$ for J1946+2300, with the zero contour suppressed. All sources appear dominated by a single compact component.
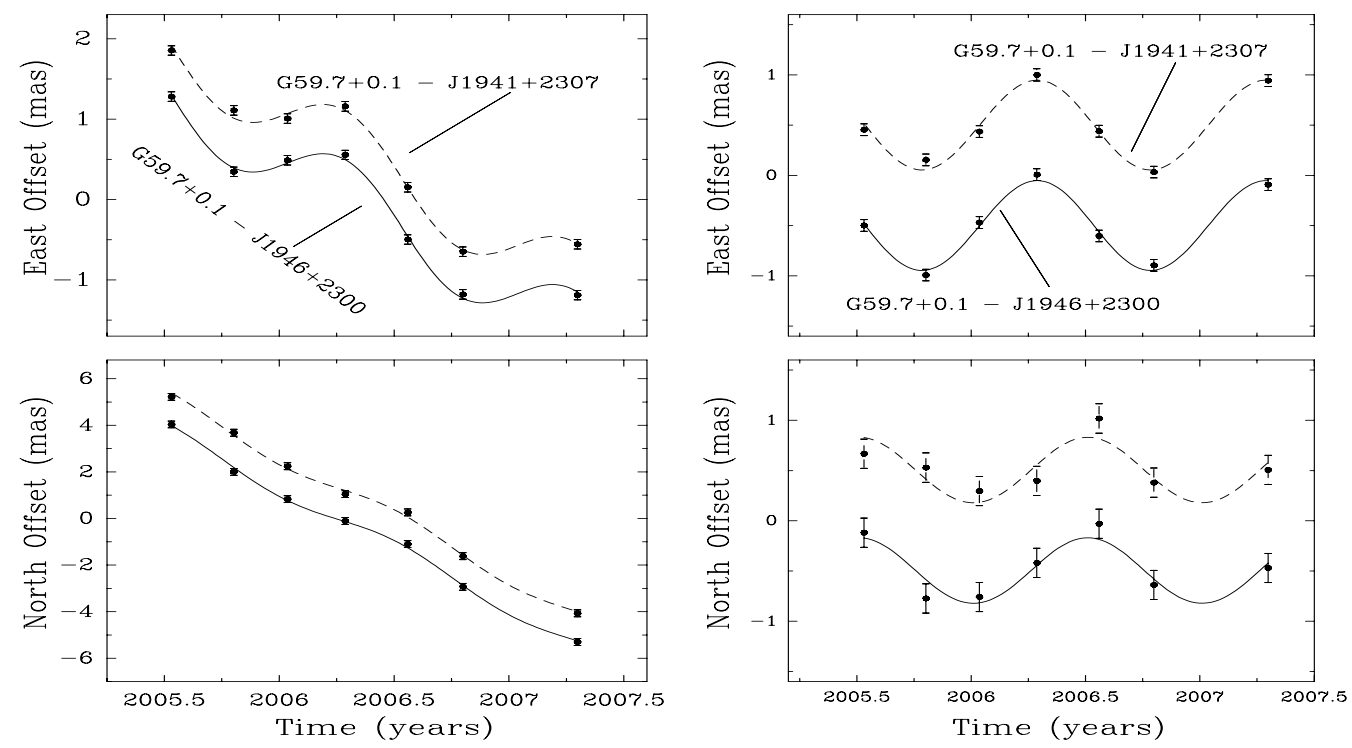

Figure 2. Position versus time for the reference maser spot at $\mathrm{V}_{L S R} 27.4 \mathrm{~km} \mathrm{~s}^{-1}$ relative to two background radio sources (offset for clarity). The left and right panels indicate the positions of the maser spot before and after removing the fitted proper motion. The top and bottom panels show the eastward and northward offsets.

Toward North Galactic Pole: $-6 \pm 0.5 \mathrm{~km} \mathrm{~s}^{-1}$.

This work is supported by NSFC grant 10673024 .

\section{References}

H. Beuther \& P. Schilke, 2004, Scince, 303, 1167

H. Beuther et al. 2002, A\&SA 390, 289

Y. Xu, M. J. Reid, X. W., \& Zheng, K. M. Menten 2006, Scince, 311, 54 